\title{
Numerical simulation of structures using generalized models for data uncertainty
}

\author{
W. Graf, J.-U. Sickert \& F. Steinigen \\ Department of Civil Engineering, Institute for Structural Analysis, \\ Technische Universität Dresden, Germany
}

\begin{abstract}
The challenging task in computational engineering is to model and predict numerically the behaviour of engineering structures in a realistic manner. Beside sophisticated computational models and numerical procedures to map physical phenomena and processes onto structural responses, an adequate description of available data covering the content of provided information is of prime importance. Generally, the availability of information in engineering practice is limited due to available resources. Far beyond the capability to specify crisp values, data are imprecise, diffuse, fluctuating, incomplete, fragmentary and frequently expert specified. Beside objective characteristics like randomness, available data are influenced by subjectivity to a considerable extend. This impedes the specification of probabilistic models with crisp parameter values to describe the uncertainty. Applying imprecise probabilities objective components of the uncertainty as well as subjective components can be considered simultaneously. A sophisticated procedure to handle imprecise probabilities provide the uncertainty model fuzzy randomness. Since fuzziness, randomness, and fuzzy randomness can be processed simultaneously, it is denoted as generalized uncertainty model. The models are demonstrated by means of a numerical example to emphasize their features and to underline their applicability.
\end{abstract}

Keywords: computational methods, numerical simulation, data uncertainty.

\section{Introduction}

The load-bearing behaviour of structures during lifetime is influenced by many static and dynamic alterations. Whether a nonlinear numerical simulation of the load-bearing behaviour leads to realistic results, depends on the quality and com- 
plexity of computational models and methods as well as the reliability of available input data. In most cases the available input data can only be reliable described as uncertain variables. Consideration of these data uncertainty in numerical analysis requires adequate computational models for processing uncertain data. Fig. 1 shows three examples for the computation of the load-bearing behaviour of structures under consideration of uncertain data. The result of loading test of a textile reinforced concrete bridge is also drawn in fig. 1a) in order to validate the numerical results. The structural behavior of an assembly of a vehicle body (fig. 1b)) of a commercial car during a crash represents a dynamic problem. As displayed in fig. 1c), also the destruction of structures by blasting can be simulated as dynamic system under consideration of data uncertainty.

a)

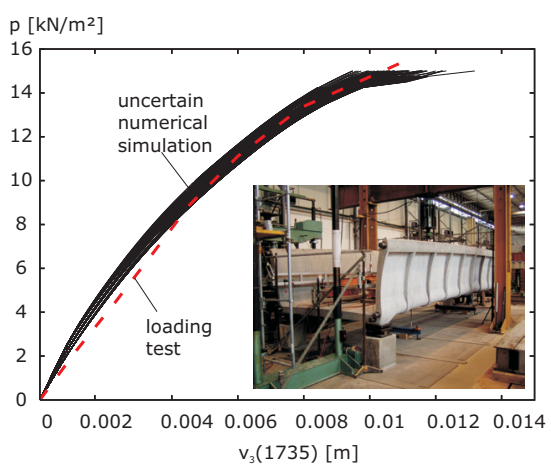

c)

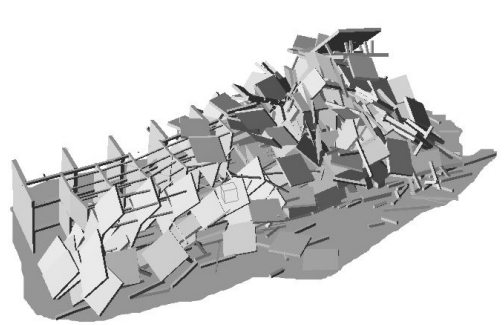

b)
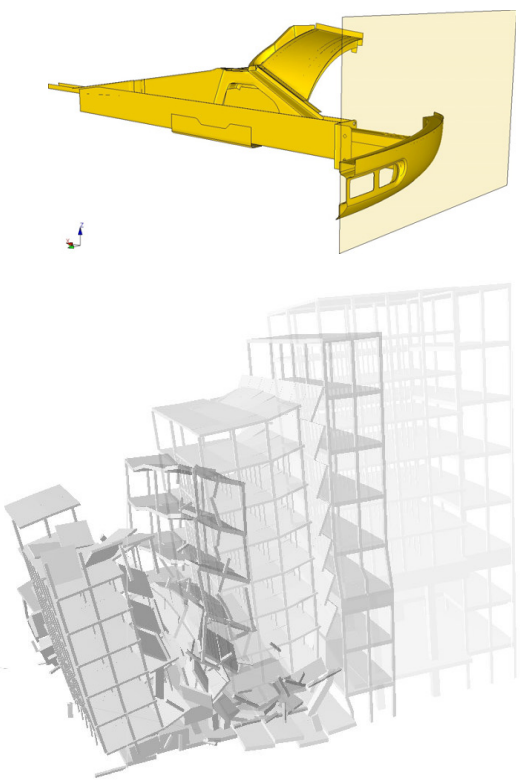

Figure 1: Fields of application of uncertain structural analysis.

\section{Modeling of uncertain variables}

The parameters - for geometry, material, load etc. - of the numerical simulations during the lifetime of structures are generally uncertain parameters. The following mathematical models are available to describe uncertainty (see also fig. 2), whereas fuzziness and randomness are considered as special cases of the generalized model fuzzy randomness [1]. The choose of the model depends on the available data. 


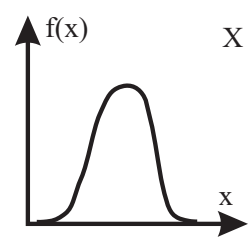

a) randomness

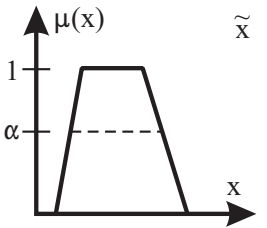

b) fuzziness

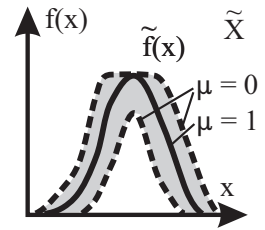

c) fuzzy randomness

Figure 2: Mathematical models of uncertainty.

The advancement of the traditional probabilistic uncertainty model enables the additional consideration of epistemic uncertainty. Thereby, epistemic uncertainty is associated with human cognition, which is not limited to a binary measure. Contrary to this, interval mathematics are limited to a binary assessment. Advanced concepts allow a gradual assessment of intervals. This extension can be realized with the uncertainty characteristic fuzziness, quantified by means of fuzzy set theory.

If sufficient statistical data exist for a parameter and the reproduction conditions are constant, the parameter may be described stochastically. Thereby the choose of the type of the probability distribution function affects the result considerably.

\subsection{Fuzzy variables}

Often the uncertainty description for parameters is based on pure expert judgment or samples which are not validated statistically. Then the description by the uncertainty model fuzziness is recommended. The model comprehends both objective and subjective information. The uncertain parameters are characterized with the aid of a membership function $\mu(x)$, see fig. 2b) and eq. (1). The membership function $\mu_{\mathrm{x}}(\mathrm{x})$ assesses the gradual membership of elements to a set. Fuzzy variables

$$
\tilde{\mathrm{x}}=\left\{\left(\mathrm{x} ; \mu_{\mathrm{x}}(\mathrm{x})\right) \mid \mathrm{x} \in X\right\} ; \quad \mu_{\mathrm{x}}(\mathrm{x}) \geq 0 \forall \mathrm{x} \in X
$$

may be utilized to describe the imprecision of structural parameters directly as well as to specify the parameters of fuzzy random variables.

\subsection{Fuzzy random variables}

If, e.g., reproduction conditions vary during the period of observation or if expert knowledge completes the statistical description of data, an adequate uncertainty quantification succeeds with fuzzy random variables. The theory of fuzzy random variables is based on the uncertainty model fuzzy randomness representing a generalized model because of it joins both stochastic and non-stochastic properties. A fuzzy random variable $\tilde{\mathrm{X}}$ is defined as the fuzzy set of their originals, whereby each original is a real-valued random variable $\mathrm{X}$. 


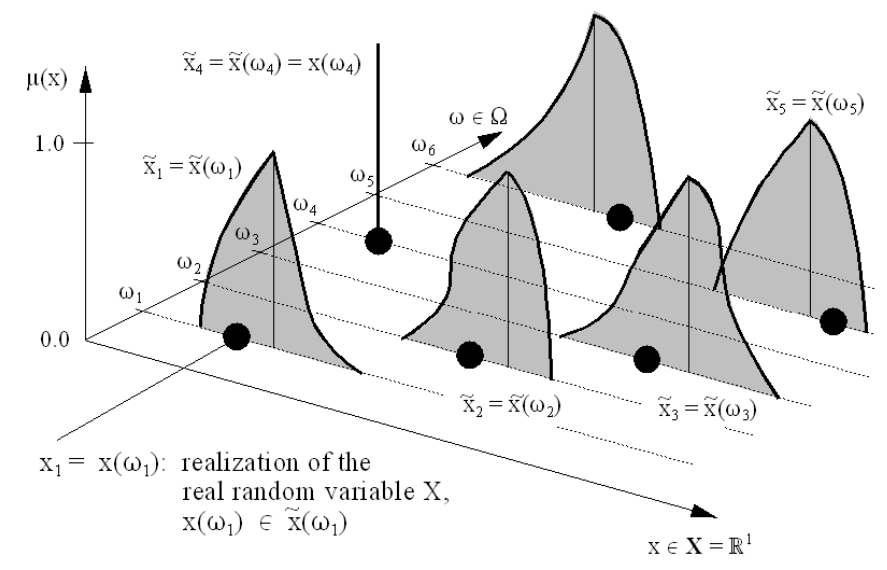

Figure 3: Fuzzy realizations of a fuzzy random variable.

The representation of fuzzy random variables presented in this paper, bases on the definition of fuzzy random variables according to [2]. The space of the random elementary events $\Omega$ is introduced. Here, e.g. the measurement of a structural parameter may be an elementary event $\omega$. Each elementary event $\omega \in \Omega$ generates not only a crisp realization like the displayed dots but a fuzzy realization $\tilde{\mathrm{x}}(\omega)=\tilde{\mathrm{x}}$, in which $\tilde{\mathrm{x}}$ is an element of the set $\mathbf{F}(\mathbb{R})$ of all fuzzy variables on $\mathbb{R}$. fig. 3 shows exemplarily five fuzzy realizations $\tilde{\mathrm{X}}$ of a fuzzy random variable $\tilde{\mathrm{X}}$. Each fuzzy variable is defined as a convex, normalized fuzzy set, whose membership function $\mu_{\mathrm{x}}(\mathrm{x})$ is at least segmentally continuous. As special case also crisp realizations as $\tilde{\mathrm{x}}_{4}=\mathrm{x}\left(\omega_{4}\right)$ may be considered. Accordingly, a fuzzy random variable $\tilde{\mathrm{X}}$ is the fuzzy result of the mapping given by

$$
\tilde{\mathrm{X}}: \Omega \mapsto \mathbf{F}(\mathbb{R})
$$

Based on this formal definition a fuzzy random variable is described by their fuzzy probability distribution function (fuzzy pdf) $\tilde{\mathrm{F}}(\mathrm{x})$. The function $\tilde{\mathrm{F}}(\mathrm{x})$ is defined as the set of real-valued probability distribution functions $F(x)$ which are gradually assessed by the membership $\mu_{F}(F(x)) . F(x)$ is the pdf of the original $X$ and is referred to as trajectory of $\tilde{\mathrm{F}}(\mathrm{x})$. As result, a fuzzy functional value $\tilde{\mathrm{F}}\left(\mathrm{x}_{\mathrm{i}}\right)$ belongs to each value $x_{i}$, see fig. 4 . Thus, $\tilde{F}(x)$ represents a fuzzy function as defined in sect. 3.1. A fuzzy probability density function is defined accordingly.

$$
\tilde{\mathrm{f}}(\mathrm{x})=\left\{\left(\mathrm{f}(\mathrm{x}) ; \mu_{\mathrm{f}}(\mathrm{f}(\mathrm{x}))\right) \mid \mathrm{f} \in \mathbf{f}\right\} ; \quad \mu_{\mathrm{f}}(\mathrm{f}(\mathrm{x})) \geq 0 \forall \mathrm{f} \in \mathbf{f}
$$





Figure 4: Fuzzy probability density and cumulative distribution function.

\section{Modeling of uncertain functions}

\subsection{Fuzzy function}

In the case that parameters depend on crisp or uncertain conditions, they are considered as fuzzy functions $\tilde{\mathrm{x}}(\underline{\tilde{\mathrm{t}}})=\tilde{\mathrm{x}}(\underline{\tilde{\theta}}, \tilde{\tau}, \underline{\tilde{\varphi}})$ or fuzzy processes $\tilde{\mathrm{x}}(\tilde{\tau})$. Variables may be the time $\tilde{\tau}$, the spatial coordinates $\underline{\tilde{\theta}}$ and further parameters $\underline{\tilde{\varphi}}$, e.g. temperature. A fuzzy function $\tilde{\mathrm{x}}(\underline{\tilde{\mathrm{t}}})$ enables the formal description of at least piecewise continuous uncertain structural parameters in $(R)$. The following definition of fuzzy functions is introduced. Given are

- the fundamental sets $\mathbf{T} \subseteq \mathbb{R}$ and $\mathbf{X} \subseteq \mathbb{R}$

- the set $\mathbf{F}(\mathbf{T})$ of all fuzzy variables $\underline{\tilde{t}}$ on the fundamental set $\mathbf{T}$

- the set $\mathbf{F}(\mathbf{X})$ of all fuzzy variables $\tilde{\mathbf{X}}$ on the fundamental set $\mathbf{X}$.

Then, the uncertain mapping of $\mathbf{F}(\mathbf{T})$ to $\mathbf{F}(\mathbf{X})$ that assigns exactly one $\tilde{\mathbf{x}} \in \mathbf{F}(\mathbf{X})$ to each $\tilde{\underline{t}} \in \mathbf{F}(\mathbf{T})$, respectively, is referred to as a fuzzy function denoted by

$$
\begin{gathered}
\tilde{\mathrm{x}}(\underline{\tilde{\mathrm{t}}}): \quad \mathbf{F}(\mathbf{T}) \stackrel{\sim}{\mapsto} \mathbf{F}(\mathbf{X}) \\
\tilde{\mathrm{x}}(\underline{\tilde{\mathrm{t}}})=\left\{\tilde{\mathrm{x}}_{\mathrm{t}}=\tilde{\mathrm{x}}(\underline{\tilde{\mathrm{t}}}) \quad \forall \underline{\tilde{\mathrm{t}}} \mid \underline{\tilde{\mathrm{t}}} \in \mathbf{F}(\mathbf{T})\right\}
\end{gathered}
$$

In fig. 5 a fuzzy process $\tilde{\mathrm{x}}(\tau)$ is presented, which assigns a fuzzy quantity $\tilde{\mathrm{x}}\left(\tau_{\mathrm{i}}\right)$ to each time $\tau_{i}$.

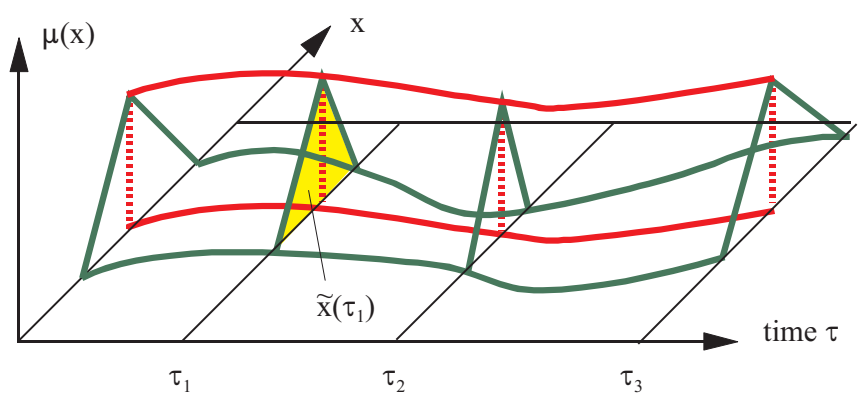

Figure 5: Fuzzy process $\tilde{\mathrm{x}}(\tau)$. 
For the numerical simulation the bunch parameter representation of a fuzzy function is applied.

$$
\tilde{\mathrm{x}}(\underline{\tilde{\mathrm{s}}}, \underline{\mathrm{t}})=\left\{\tilde{\mathrm{x}}_{\mathrm{t}}=\tilde{\mathrm{x}}(\underline{\tilde{\mathrm{s}}}, \underline{\mathrm{t}}) \quad \forall \underline{\mathrm{t}} \mid \underline{\mathrm{t}} \in \mathbf{T}\right\}
$$

For each crisp bunch parameter vector $\underline{\mathrm{s}} \in \underline{\tilde{\mathrm{s}}}$ with the assigned membership value $\mu(\underline{\mathrm{s}})$ a crisp function $\mathrm{x}(\underline{\mathrm{t}})=\mathrm{x}(\underline{\mathrm{s}}, \underline{\mathrm{t}}) \in \tilde{\mathrm{x}}(\underline{\mathrm{t}})$ with $\mu(\mathrm{x}(\underline{\mathrm{t}}))=\mu(\underline{\mathrm{s}})$ is obtained. The fuzzy function $\tilde{\mathrm{x}}(\underline{\mathrm{t}})$ may thus be represented by the fuzzy set of all real valued functions $\mathrm{x}(\mathrm{t}) \in \tilde{\mathrm{x}}(\underline{\mathrm{t}})$ with $\mu(\mathrm{x}(\underline{\mathrm{t}}))=\mu(\mathrm{x}(\underline{\mathrm{s}}, \underline{\mathrm{t}}))=\mu(\underline{\mathrm{s}})$

$$
\tilde{\mathrm{x}}(\underline{\mathrm{t}})=\tilde{\mathrm{x}}(\underline{\tilde{\mathrm{s}}}, \underline{\mathrm{t}})=\{(\mathrm{x}(\underline{\mathrm{s}}, \underline{\mathrm{t}}), \mu(\mathrm{x}(\underline{\mathrm{s}}, \underline{\mathrm{t}})))|\mu(\mathrm{x}(\underline{\mathrm{s}}, \underline{\mathrm{t}}))=\mu(\underline{\mathrm{s}}) \forall \underline{\mathrm{s}}| \underline{\mathrm{s}} \in \underline{\tilde{\mathrm{s}}}\}
$$

which may be generated from all possible real vectors $\underline{\mathrm{s}} \in \underline{\tilde{\mathrm{s}}}$. For every $\underline{\mathrm{t}} \in \mathbf{T}$ each of the crisp functions $\mathrm{x}(\underline{\mathrm{t}})$ takes values which are simultaneously contained in the associated fuzzy functional values $\tilde{\mathrm{x}}(\underline{\mathrm{t}})$. The real functions $\mathrm{x}(\underline{\mathrm{t}})$ of $\tilde{\mathrm{x}}(\underline{\mathrm{t}})$ are defined for all $\underline{\mathrm{t}} \in \mathbf{T}$ referred to as trajectories. Numerical processing of fuzzy functions $\tilde{\mathrm{x}}(\underline{\mathrm{t}})=\mathrm{x}(\underline{\tilde{\mathrm{s}}}, \underline{\mathrm{t}})$ demands the discretization of their arguments $\underline{\mathrm{t}}$ in space and time.

\subsection{Fuzzy random function}

According to eqs. (2) and (4) a fuzzy random function is the result of the uncertain mapping

$$
\tilde{\mathrm{X}}(\underline{\mathrm{t}}): \mathbf{F}(\mathbf{T}) \times \Omega \rightarrow \mathbf{F}(\mathbb{R})
$$

Thereby, $\mathbf{F}(\mathbf{X})$ and $\mathbf{F}(\mathbf{T})$ denote the sets of all fuzzy variables in $\mathbf{X}$ and $\mathbf{T}$ respectively [4]. At a specific point $\underline{t}$ the mapping of eq. (8) leads to the fuzzy random variable $\tilde{\mathrm{X}}(\underline{\mathrm{t}})=\tilde{\mathrm{X}}(\underline{\mathrm{t}})$. Therefore, fuzzy random functions are defined as a family of fuzzy random variables $\tilde{\mathrm{X}}_{\mathrm{t}}$.

$$
\tilde{\mathrm{X}}(\underline{\mathrm{t}})=\left\{\tilde{\mathrm{X}}_{\mathrm{t}}=\tilde{\mathrm{X}}(\underline{\mathrm{t}}) \forall \underline{\mathrm{t}} \mid \underline{\mathrm{t}} \in \mathbf{T}\right\}
$$

For the numerical simulation again the bunch parameter representation of a fuzzy random function is applied. For each crisp bunch parameter vector $\underline{\mathrm{s}} \in \underline{\tilde{\mathrm{S}}}$ with the assigned membership value $\mu(\underline{\mathrm{s}})$ a real random function $\mathrm{X}(\underline{\mathrm{t}})=\mathrm{X}(\underline{\mathrm{s}}, \underline{\mathrm{t}}) \in \tilde{\mathrm{X}}(\underline{\mathrm{t}})$ with $\mu(\mathrm{X}(\underline{\mathrm{t}}))=\mu(\underline{\mathrm{s}})$ is obtained. The fuzzy random function $\tilde{\mathrm{X}}(\underline{\mathrm{t}})$ may thus be represented by the fuzzy set of all real random functions $\mathrm{X}(\underline{\mathrm{t}}) \in \tilde{\mathrm{X}}(\underline{\mathrm{t}})$

$$
\mathrm{X}(\underline{\tilde{\mathrm{s}}}, \underline{\mathrm{t}})=\{(\mathrm{X}(\underline{\mathrm{t}}), \mu(\mathrm{X}(\underline{\mathrm{t}})))|\mathrm{X}(\underline{\mathrm{t}})=\mathrm{X}(\underline{\mathrm{s}}, \underline{\mathrm{t}}) ; \mu(\mathrm{X}(\underline{\mathrm{t}}))=\mu(\underline{\mathrm{s}}) \forall \underline{\mathrm{s}}| \underline{\mathrm{s}} \in \underline{\tilde{\mathrm{s}}}\}
$$

which may be generated from all possible real vectors $\underline{\underline{s}} \in \underline{\tilde{\mathrm{s}}}$. Thereby, every $\underline{\mathrm{t}} \in \mathbf{T}$ is simultaneously contained in the associated fuzzy random function $\tilde{\mathrm{X}}(\underline{\mathrm{t}})$. The real random function $\mathrm{X}(\underline{\mathrm{t}}) \in \tilde{\mathrm{X}}(\underline{\mathrm{t}})$ is defined for all $\underline{\mathrm{t}} \in \mathbf{T}$ and referred to as trajectory. A numerical processing of a fuzzy random function $\tilde{X}(\underline{\mathrm{t}})=X(\underline{\tilde{\mathrm{s}}}, \underline{\mathrm{t}})$ requires the discretization of their arguments $\underline{\mathrm{t}}$ in space and time. 


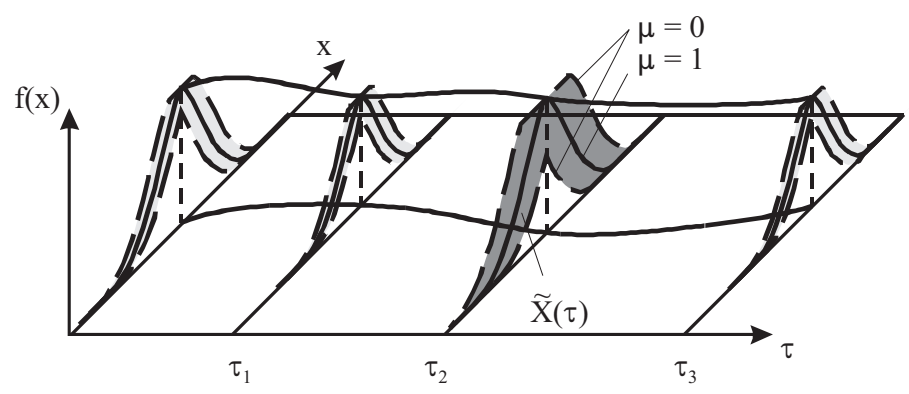

Figure 6: Fuzzy random process $\tilde{\mathrm{X}}\left(\underline{\theta}_{\mathrm{j}}, \tau\right)$.

\section{Fuzzy stochastic analysis}

Fuzzy stochastic analysis is an appropriate computational model for processing uncertain data using the uncertainty model fuzzy randomness. Basic terms and definitions related to fuzzy randomness have been introduced, inter alia, by [2]. The formal description of fuzzy randomness chosen by these authors is however not suitable for formulating uncertainty encountered in engineering problems. A suitable form of representation with the scope of numerical engineering problems is given with the so-called $\alpha$-discretization by [1] and [3].

The numerical simulation under consideration of fuzzy variables and fuzzy functions (fuzzy analysis) may formally be described by the mapping

$$
\mathrm{M}(\underline{\mathrm{t}}): \quad \underline{\tilde{\mathrm{x}}}(\underline{\mathrm{t}}) \mapsto \underline{\tilde{z}}(\underline{\mathrm{t}})
$$

According to eq. (11) the fuzzy variables $\underline{\tilde{x}}$ and the fuzzy functions $\underline{\tilde{x}}(\underline{t})$ are mapped to the fuzzy results $\underline{\tilde{z}}(\underline{\mathrm{t}})$ with aid of the crisp analysis algorithm $\mathrm{M}(\underline{\mathrm{t}})$. Every arbitrary deterministic fundamental solution may be used as algorithm $\mathrm{M}(\underline{\mathrm{t}})$. On the basis of point and time discretization, fuzzy functional values of the function $\mathrm{x}(\underline{\tilde{\mathrm{s}}}, \underline{\theta}, \tau, \underline{\varphi})$ are determined at points in space $\underline{\theta}_{\mathrm{j}}$, time $\tau_{\mathrm{i}}$, and parameters $\underline{\varphi}$.

The numerical simulation is carried out with the aid of the $\alpha$-level optimization [3]. For the fuzzy variable $\tilde{a}_{1}$ and the fuzzy function $\mathrm{x}(\underline{\tilde{\mathrm{s}}}, \underline{\theta}, \tau$ the input subspace $\underline{\mathrm{E}}_{\alpha}$ assigned to the level $\alpha$ is formed. By applying the mapping model $\mathrm{M}\left(\tau_{\mathrm{i}}\right)$ the extreme values $\mathrm{z}_{\alpha, 1}\left(\underline{\theta}_{\mathrm{j}}, \tau_{\mathrm{i}}\right)$ and $\mathrm{z}_{\alpha, \mathrm{r}}\left(\underline{\theta}_{\mathrm{j}}, \tau_{\mathrm{i}}\right)$ of the fuzzy result variable $\tilde{\mathrm{z}}\left(\underline{\theta}_{\mathrm{j}}, \tau_{\mathrm{i}}\right)$ are computed. The points are interval bounds of the $\alpha$-level sets and enable the numerical description of the convex membership function of the fuzzy result variable $\tilde{z}\left(\underline{\theta}_{j}, \tau_{i}\right)$. For the computation of $\tilde{z}\left(\underline{\theta}_{j}, \tau_{i+1}\right)$ at the time point $\tau_{i+1}$ the procedure must be restarted at $\tau=0$ due to the interaction within the mapping model.

Fuzzy stochastic analysis allows the mapping of fuzzy random input variables onto fuzzy random result variables. In the field of engineering fuzzy stochastic analysis can be applied for static and dynamic structural analysis and for assessment of structural safety, durability as well as robustness. Two different approaches for computation of the fuzzy random result variables have been developed. The first variant (fig. 7) bases on the bunch parameter representation of fuzzy random 
variables by [4]. The second variant utilizes the $l_{\alpha} \mathrm{r}_{\alpha}$-representation of fuzzy random variables. The variant to be preferred depends on the engineering problem, the available uncertain data, and the wanted results [5].

\begin{tabular}{|l|ll|}
\hline F $_{\text {FA }}\left(\mathbf{F}_{\text {SA }}(\mathbf{d})\right)$ & Fuzzy analysis \\
\cline { 2 - 3 } & $\begin{array}{r}\text { FA }_{\text {SA }}(\mathbf{d}) \\
\text { d }\end{array}$ & Stochastic analysis \\
\hline
\end{tabular}

Figure 7: Fuzzy stochastic analysis (FSA).

\section{Numerical example}

The numerical simulation is applied to a T-beam floor construction. A section of the T-beam floor construction with two beams is shown in fig. 8. The timedependent reliability in relation to serviceability limit state is computed with the aid of FSA. The serviceability limit state is defined with the maximum displacement of $\mathrm{v}_{3}=3.0 \mathrm{~cm}$ in the middle of the beam. Both fuzzy random and real random input variables are taken into consideration.
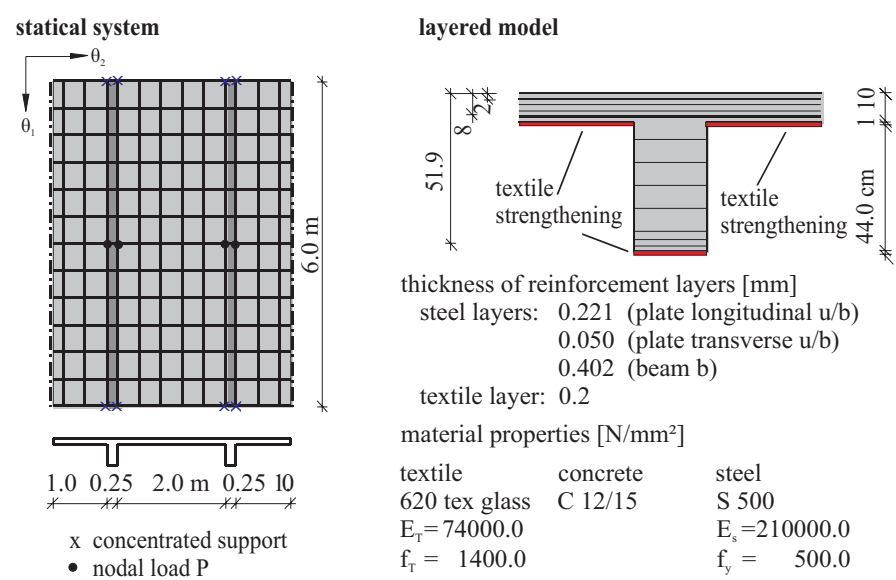

Figure 8: Geometry, material, and FE model.

For the deterministic computational analysis of the FSA, the physically nonlinear analysis with hybrid finite folded plate elements is applied. RC structures 
with textile strengthening are described appropriately with the multi-referenceplane model (MRM). The MRM is utilized to describe multi-layered composite materials with a discontinuous multi-Bernoulli-kinematics [6]. A MRM element comprises $\mathrm{k}+1$ layered sub-elements and $\mathrm{k}$ interfaces. The sub-element $\mathrm{i}$ with its corresponding reference plane $\mathrm{RP}_{\mathrm{i}}(\mathrm{i}=0, \ldots, \mathrm{k})$ is subdivided into $\mathrm{s}_{\mathrm{i}}$ sub-layers (concrete and steel sub-layers or fine-grained concrete and textile sub-layers). In order to describe the composite structure comprised of reinforced concrete and textile strengthening, different nonlinear material laws are applied to the individual sub-layers of concrete, steel, and textile. Endochronic material laws for concrete and steel are utilized for general loading, unloading, and cyclic loading processes, and taking into account the accumulated material damage during the load history. In the case of cyclic loading, the textile-reinforced fine-grained concrete layers are split into sub-layers of fine-grained concrete and of textile reinforcement. The endochronic material law for concrete is adapted to the fine-grained concrete. A nonlinear elastic-brittle material law is used for the textile reinforcement.

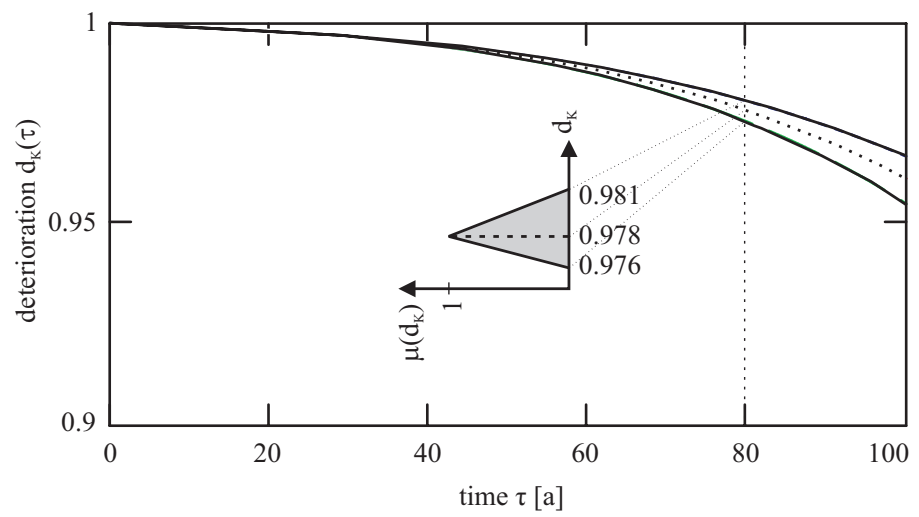

Figure 9: Fuzzy process $\tilde{\mathrm{d}}_{\mathrm{K}}$.

The structure is discretized by means of 156 MRM elements. The beams are modeled with 12 concrete layers and the plate part of the floor is using five concrete layers. The steel reinforcement is specified as an uniaxial smeared layer in each case. Crack formation, tension stiffening, and steel yielding are taken into consideration. The T-beam floor was designed for a dead weight $\mathrm{g}$ of the floor construction, an additional load $g_{1}$, and a live load $\mathrm{p}_{1}$. Due to a conversion, it is necessary to dimension the floor additionally for a live load $\mathrm{p}_{2}$ and point loads P. For this reason textile strengthening is applied to the underside of the construction (fig. 8). The loads $\mathrm{g}_{1}, \mathrm{p}_{1}$, and $\mathrm{p}_{2}$ are modeled as uniformly distributed superficial loads. The point loads $\mathrm{P}$ are modeled as nodal loads of magnitude $\mathrm{P}=30 \mathrm{kN}$. The live load $\mathrm{p}_{1}$ is modeled as a real random variable (Gumbel distribution with $\mathrm{a}=2.565$ and $\mathrm{b}=$ 5.699) and the live load $\mathrm{p}_{2}$ as fuzzy random variable (Gumbel distribution with the bunch parameters $\sigma_{\mathrm{p}_{2}}=0.5 \mathrm{kN} / \mathrm{m}^{2}$ and $\left.\tilde{\mathrm{E}}_{\mathrm{p}_{2}}=<5.7,6.0,6.3>\mathrm{kN} / \mathrm{m}^{2}\right)$. The 
concrete compressive strength $\beta_{\mathrm{c}}$ is specified in this case as a Gaussian distributed fuzzy random variable $\left(\tilde{\sigma}_{\beta_{c}}=<2.0,2.5,3.0>\mathrm{N} / \mathrm{mm}^{2}\right.$ and $\left.\mathrm{E}_{\beta_{\mathrm{c}}}=20 \mathrm{~N} / \mathrm{mm}^{2}\right)$. The fine-grained concrete tensile stress $\sigma_{\mathrm{t}}=(0.3+\mathrm{c}) \beta_{\mathrm{fgc}}^{2 / 3}$ is modeled as random variable by means of the Gaussian distributed parameter $\mathrm{c}$ with $\mathrm{E}_{\mathrm{c}}=0$ and $\sigma_{\mathrm{c}}=0.01$.

The time-dependent reliability in relation to serviceability limit state requires the consideration of deteriorating effects. The deterioration is influenced by several factors, which are not precisely known [7]. This information deficit leads to uncertainty and at the end to an uncertain service life. The uncertain deterioration is simplified by the fuzzy process $\tilde{\mathrm{d}}_{\mathrm{K}}=\mathrm{e}^{-\int \chi(\tau, \mathrm{s}) \mathrm{d} \tau}$ which acts on the global stiffness matrix, see fig. 9. The processes $\chi(\tau, \mathrm{s})$ in this example are determined as $\chi(\tau, \mathrm{s})=0.0001$ if $\tau \leq \tau_{0}$, and $0.0001+1 / 6000\left(\mathrm{e}^{0.02 \cdot \tau \cdot \mathrm{s}}-\mathrm{e}^{\left.0.02 \cdot \tau_{0} \cdot \mathrm{s}\right)}\right.$ if $\tau>0$ with $\tau_{0}=20$ years and the bunch parameter $\mathrm{s} \in \tilde{\mathrm{s}}=<0.9,1.0,1.1>$, see fig. 9 . As results of the uncertain numerical simulation, fig. 10 shows the time-dependent fuzzy reliability.

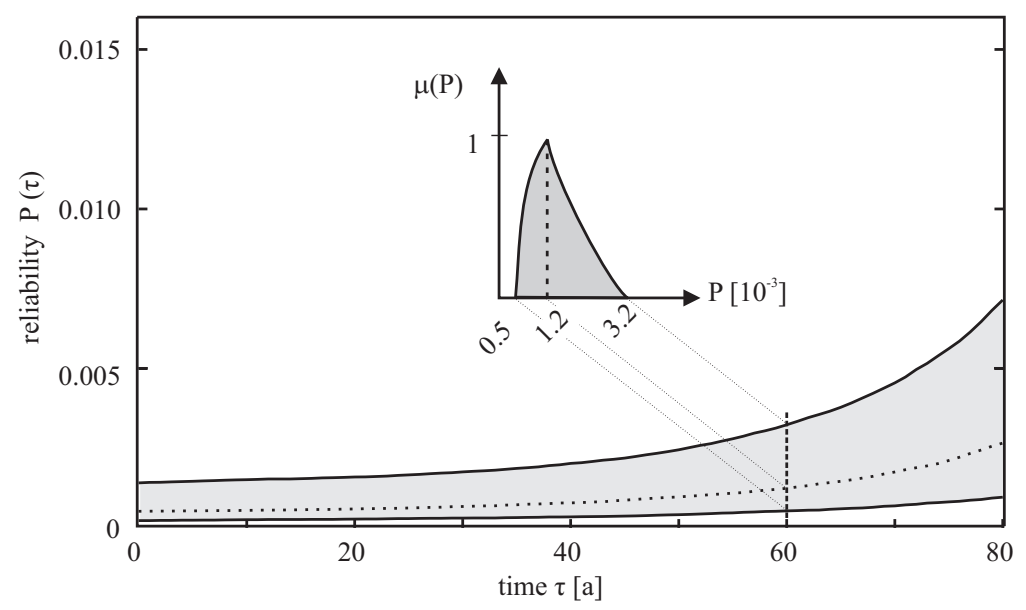

Figure 10: Time-dependent fuzzy reliability.

\section{Acknowledgement}

The authors gratefully acknowledge the support of the German Research Foundation.

\section{References}

[1] Möller, B.; Beer, M. Fuzzy Randomness. Uncertainty in Civil Engineering and Computational Mechanics, Springer: Berlin, Heidelberg, 2004. 
[2] Puri, M.L.; Ralescu, D. Fuzzy random variables. Journal of Mathematical Analysis and Applications, 114 (1986), pp. 409-422.

[3] Möller, B.; Graf, W.; Beer, M. Fuzzy structural analysis using $\alpha$-level optimization, Computational Mechanics, 26(2000), pp. 547-565.

[4] Sickert, J.-U.; Beer, M.; Graf, W.; Möller, B. Fuzzy probabilistic structural analysis considering fuzzy random functions. In: 9th International Conference on Applications of Statistics and Probability in Civil Engineering, Rotterdam, Millpress 2003, pp. 379-386.

[5] Möller, B.; Graf, W.; Sickert, J.-U.; Reuter, U. Numerical simulation based on fuzzy stochastic analysis, Mathematical and Computer Modelling of Dynamical Systems (MCMDS), Taylor \& Francis, 13(2007) 4, pp. 349-364.

[6] Möller, B.; Graf, W.; Hoffmann, A.; Steinigen, F. Numerical simulation of structures with textile reinforcement, Computers and Structures, 83(2005), pp. 1659-1688.

[7] Möller, B.; Graf, W.; Sickert, J.-U.; Beer, M. Time-dependent reliability of textile strengthened RC structures under consideration of fuzzy randomness, Computers and Structures, 84(2006), pp. 585-603. 